\title{
Production and quality control policies for deteriorating manufacturing system
}

\author{
HÉCTOR RIVERA-GOMEZ ${ }^{1}$, Ali GHARBI ${ }^{1}$, JEAN PIERRE KENNÉ ${ }^{2}$ \\ ${ }^{1}$ Automated Production Engineering Department, École de Technologie Supérieure, \\ Production System Design and Control Laboratory, Université du Québec \\ 1100 Notre Dame Street West, Montreal, QC, Canada, H3C 1K3 \\ hriver06@hotmail.com \\ ali.gharbi@etsmtl.ca \\ ${ }^{2}$ Mechanical Engineering Department, École de Technologie Supérieure, \\ Laboratory of Integrated Production Technologies, Université du Québec \\ 1100 Notre Dame Street West, Montreal, QC, Canada, H3C 1K3 \\ jean-pierre.kenne@etsmtl.ca
}

\begin{abstract}
In this paper, a novel model for a deteriorating manufacturing system is analyzed, considering repairs and overhauls of random durations. The machine manufactures one product and the model is further complicated because the quality of the parts' produced deteriorates according to the wear of the machine and human interventions. When a breakdown occurs, either a repair or an overhaul is performed. The machine is restored to as-good-as-new conditions if the overhaul is selected, and conversely, its condition deteriorates following repairs. Multiple operational states are considered to define an aging process. The decision variables of the model are the production rate and the repair/overhaul switching strategy. This paper provides new insights to this research area by considering the simultaneous production and repair/overhaul control policy under the effect of deteriorations. The optimal decision policy minimizes the total incurred cost comprising the inventory, backlog, repair and overhaul costs over an infinite planning horizon. Our paper differs from other research projects in its consideration of the machine's history, defined by the number of repairs and multiple operational states. A numerical example is given to illustrate the proposed approach and a sensitivity analysis has been conducted to confirm the structure of the obtained control policies.
\end{abstract}

Keywords: Quality, Manufacturing system, Optimal control, Numerical methods, Deteriorating systems. 


\section{Introduction}

In the area of manufacturing systems, quality is one of the most important factors that define the market survival of a company. Moreover, the quality of a manufacturing system may be affected by the deterioration caused by a series of events, such as breakdowns, repairs, wear, fatigue, corrosion, human errors, etc. Despite the importance of quality, only a few research works have included quality issues in the determination of control policies. These considerations strongly motivate the need of an integrated model that allows us to examine the effect of the inter-relation between production planning and quality issues. Nevertheless other situations may arise, for instance the manufacturing system can be subject to deteriorations, and so at including this factor it is needed further research to have a better understanding of the production system behavior.

The production planning problem of manufacturing systems started attracting increased attention with the work of Akella and Kumar (1986). They proposed an analytical solution to the problem that consisted in controlling the production rate of a failure-prone manufacturing system. This model was extended by Bielecki and Kumar (1988), when they provided another solution for a similar production system. These works helped to consolidate the concept of the so-called Hedging Point Policy (HPP). Later, Sharifnia (1988) established that the HPP is susceptible to generalizations, such as the multi-state property that is used in subsequent works. Over the years, the hedging point policy has grown in complexity; for instance, Gharbi and Kenne (2003) studied the problem of production control for a production system which involves multiple machines producing different part types. Other works, such as Chelbi and Ait-Kadi (2004), have focused on the joint strategy of buffer stock production and preventive maintenance. It has been followed other extensions treating a wide range of aspects such as preventive maintenance as in Rezg et al. (2008), unreliable suppliers as in Hajji et al. (2009) and multi-products production plan as in Dahane et al. (2012). However, it is observed from these extensions that a significant branch of the literature has as main assumption that the manufacturing system produces only conforming parts. Unfortunately, in an industrial context, this assumption is incomplete. The above mentioned papers have therefore not treated the interaction between production and quality issues. This argument led us to develop an integrated model in which the aspects of production and quality are considered, and their interaction is examined.

A limited number of authors have addressed quality issues and the interaction of quality with production planning. The need to include quality in the design of production lines was identified by Inman et al. (2003), who presented several classes of decisions that affect quality and productivity. A comprehensive work on this interaction is the series of papers by Kim and Gershwin (2005, 2008), who mathematically analyzed the performance of manufacturing systems with quality aspects. Also, they extended the mathematical approximation method, called decomposition, to evaluate the performance of transfer lines incorporating the effect of quality. The decomposition method has been extensively applied to analyzed long production systems as in Bonvik et al. (2000). In the same direction, the set of works of Colledani and Tolio $(2006,2009,2011)$ presented a 
mathematical method based on discrete Markov chains to evaluate the performance of production systems with manufacturing and inspection machines, where the behavior of the machines were monitored with control charts. Nevertheless, the main difference between these works and our paper is that in the former, Markov chains and the decomposition technique are the main tools used in their analysis, whereas in this paper, we focus on the structure of the control policy derived from a semi-Markov model. Other applications tackle the effect of quality to determine maintenance actions, such as in Radhoui et al. (2009), where they considered production in batches, and used the rejection rate and the buffer size as the decision variables to determine maintenance activities. The maintenance strategy has also been covered by Njike et al. (2009), who developed a model to simultaneously control maintenance activities and production planning, and considered several operational states that monitor the system's health. The idea was to use the quantity of defective products as feedback to optimally control the system. In spite of the above-cited authors, our paper contributes to this growing research area by considering that it is plausible to combine the production planning problem with deterioration in a different direction. While the literature contains many proposals that model deterioration, in our research, however, we conjecture that the manufacturing system is subject to a degrading process, and that this phenomenon is tied directly to the quality of the parts produced. We find some interesting ideas to support this assumption, in the area of deteriorating systems.

Deteriorating systems have been studied by several authors, who have determined optimal policies based on either the age of the machine or its accumulated number of failures. For example, in the series of works presented by Love et al. $(1998,2000)$, it is considered that at failure, the machine may undergo a repair that partially resets its failure intensity, or be put through a second option, which is to conduct a major repair that restores the machine to an as-good-as-new condition. This model was extended by Dehayem et al. (2011a), who included production planning in the repair/replacement problem. They considered imperfect repair actions, in which the repair time increases with the number of failures and the machine is also age-deteriorated. Lather the authors included preventive maintenance in their model in Dehayem et al. (2011b). The main observation regarding these papers is that, they relate the concept of deterioration with either the time to failure or the repair time, and therefore do not examine the effect of deterioration on the rate of defectives. In contrast, in our research, we want to focus on the interaction between deterioration and the quality of the parts produced. To model the quality-deterioration, in this paper we propose that it is given by the combined effect of two factors; the wear of the machine and human interventions. For this reason we define an internal dynamic for the operational state, which leads to the use of multiple operational states that reflect different quality levels. Furthermore, we complement the deterioration modeling with the consideration of human interventions represented by worse repairs (a maintenance action which makes the rate of defectives increase). We did not find available work in the literature with this approach. The quality deterioration is countered by a major overhaul that restores the production system to initial conditions.

The aforementioned issues are therefore considered in this paper where we present a new model for the simultaneous determination of the production and the repair/overhaul switching policies for a manufacturing system whose produced parts' quality deteriorates 
over time. The model falls under the class of machine rate of defectives dependent models that aims to incorporate production and quality issues in an integrated model. The optimization problem consists in the joint determination of the production rate and repair/overhaul switching policies in a stochastic environment, where the following considerations are included in the model:

a) The rate of defectives increases with the number of repairs, and so the machine repair's history is needed to model more realistically the deterioration phenomenon and optimally control the manufacturing system.

b) Between failures, there is an aging process defined by multiple operational states that model different quality yields. Where each operational state models a different rate of defectives.

This paper differs from other research projects in that here, due to the degrading process, the production rate and repair/overhaul switching strategy are determined simultaneously with a semi-Markov process, something which has not been yet addressed in the published literature. It is important to note that in this case, Markovian models are not appropriate since the quality of the parts produced, defined by the rate of defectives, depends on the history of the machine. In our model, the machine's history is related to the number of repairs and the set of operational states. The production and repair/overhaul switching policies are determined to minimize the inventory, backlog, repair and overhaul costs over an infinite planning horizon.

The rest of the paper is organized as follows. After an overview of the literature in section 1, we present the notations, the problem statement and the mathematical formulation of the problem in section 2, also optimality conditions and the numerical approach applied are defined in this section. We present a numerical example to identify the control policy of the problem as well as the respective control factors in section 3. An example of an implementation of the obtained results is outlined in section 4. Discussions on the behaviors of the production system based on a sensitivity analysis are provided in section 5. The paper is concluded in section 6 .

\section{Notation and problem statement}

This section presents the notation, the problem statement and the formulation of the control problem.

\subsection{Notation}

The following notations are used in this paper:

$\begin{array}{ll}x(t) & \text { Inventory level at time } t \\ u(t) & \text { Production rate of the manufacturing system at time } t \\ n(t) & \text { Current number of failures at time } t \\ d & \text { Constant demand rate } \\ \xi(t) & \text { Mode of the machine at time } t \\ U_{\max } & \text { Maximum production rate }\end{array}$




$\begin{array}{ll}\beta(\cdot) & \text { Rate of defectives } \\ \rho & \text { Discount rate } \\ \pi_{i} & \text { Limiting probability at mode } i \\ J(\cdot) & \text { Expected discounted cost function } \\ \lambda_{\alpha \alpha} \cdot(\cdot) & \text { Transition rate form mode } \alpha \text { to mode } \alpha \\ \omega(\cdot) & \text { Control variable for the repair/overhaul policy } \\ \omega_{\min } & \text { Minimum value for the control variable } \omega \\ \omega_{\max } & \text { Maximum value of the control variable } \omega \\ g(\cdot) & \text { Cost rate function } \\ v(\cdot) & \text { Value function } \\ \tau & \text { Jump time of } \xi(t) \\ c^{+} & \text {Incurred cost per unit of produced parts for positive inventory } \\ c^{-} & \text {Incurred cost per unit of produced parts for backlog } \\ c_{r} & \text { Worse repair cost } \\ c_{o} & \text { Overhaul cost } \\ O P_{i}^{n} & \text { Operational state } i \text { at the } \mathrm{n}^{\text {th }} \text { failure } \\ S & \text { Number of operational states of the aging process for any number } \\ & \text { of repairs } n \\ N & \text { Maximum number of failures during which the system remains } \\ & \text { operational }\end{array}$

\subsection{Problem statement}

The manufacturing system under study consists of an unreliable single machine producing one part type. The machine is subject to random events, such as failures and maintenance activities; it can produce at maximum capacity or at demand rate to satisfy a constant demand of product. Figure 1 presents the block diagram of the production system. Since the machine, as illustrated in (a) in Figure 1, is unreliable, there is a buffer stock, as shown in (b), to counter the effect of failures. However, the quality of the products produced is not perfect, as it exists a certain percentage of defectives. The stock is thus a mixture of flawless and defective products. Moreover, we propose that the machine experience a quality deterioration phenomenon, depicted by (c), which leads to an increasing rate of defectives $\beta$. It should be noted though, that in the area of deteriorating systems, the effect of deterioration can be manifested either through increasing repair times or decreasing times to failure. In this paper, we focus on the effect of deterioration on the quality of the parts produced. To model this condition, we propose that deterioration is a combination of: i) an aging process, where quality deteriorates because of the impact of the natural wear of the machine: ii) worse repairs, in which case quality deteriorates due to the influence of human interventions, represented by repairs that leave the machine in a worse condition than before repair. The control policy of the model, represented by (d), implies decision variables related to the production planning and quality control defined by the repair/overhaul strategy. This policy copes with the deterioration phenomenon. When the machine fails, the decision maker has two options:

1) Perform an expensive and time-consuming repair called overhaul, which restores the rate of defectives to initial conditions, or 
2) Carry out an inexpensive worse repair which lets the machine operate for a while, but with the disadvantage that it deteriorates the machine, increasing the rate of defectives.

We intend to determine the control policy (production rate and the repair/overhaul switching strategy) that minimizes the average total cost, composed of the inventory cost, the backlog cost, the repair and overhaul cost.

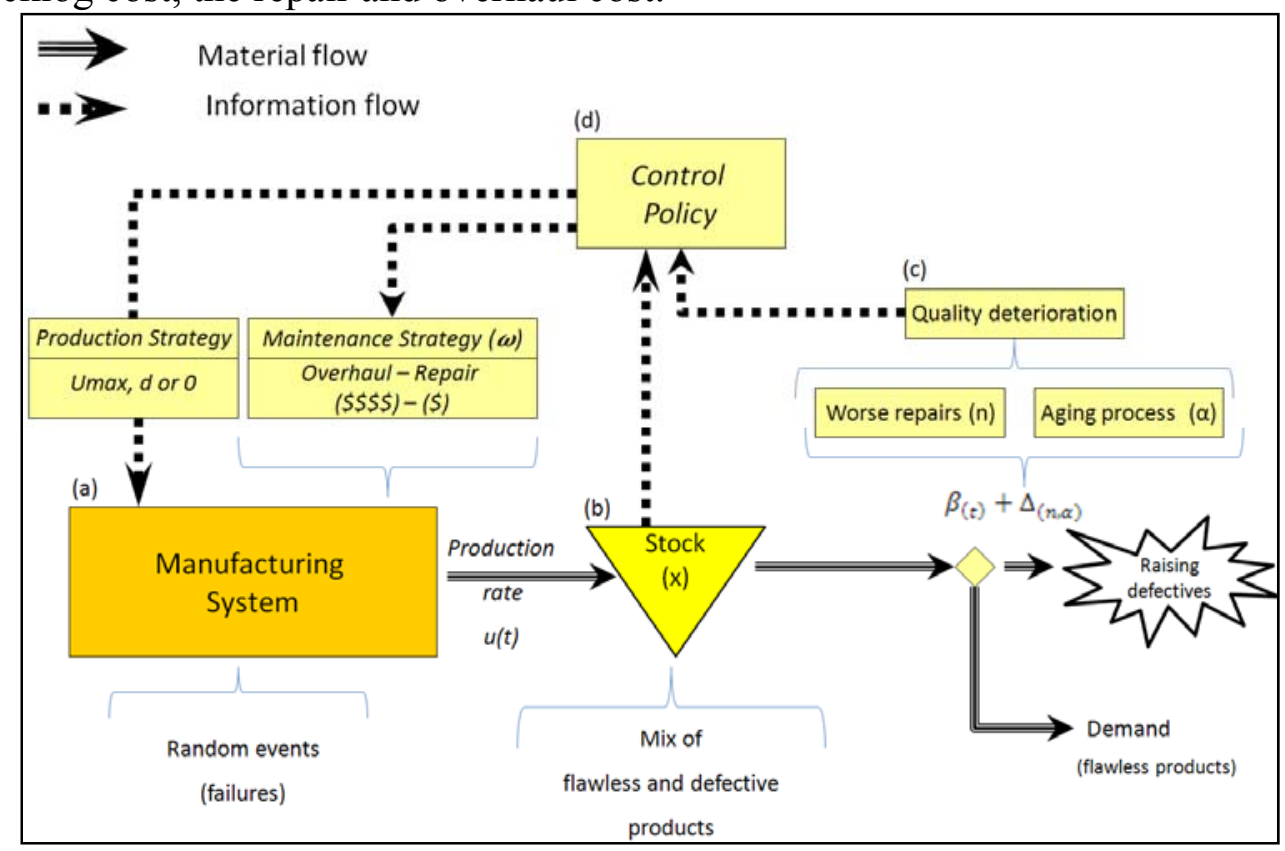

Figure 1: Block diagram of the manufacturing system under study

\subsection{Formulation of the control problem}

We shall begin the formulation of the control model by presenting the transition diagram of the manufacturing system in Figure 2. We emphasize that the machine is subject to deterioration, and that this has a strong effect on the rate of defectives. To model the quality deterioration phenomenon, we used the concept of the aging process, as illustrated in (A) in Figure 2. The fact is that the aging process models the natural wear of the machine. To that end, we propose multiple operational states, as depicted in (B) that reflect different quality yields. The operational states $O P_{i}^{n}$ are divided into several stages, with the quality of the parts produced deteriorating over time as the machine moves in the operational states. This means that the rate of defectives $\beta$ increases in every operational state. Furthermore, the machine is subject to random failures. When it is in the failure state $F_{\mathrm{n}}$ it implies a decision point, as presented in (C), where two types of actions can be taken. An expensive repair called overhaul can be performed, as in (D), which counters the effect of the quality deterioration. The second option is to perform a worse repair, as shown in (E), which is less expensive and faster than the first option, but which deteriorates the machine's conditions; specifically it increases the rate of defectives. In fact, the quality deterioration modeling is complemented by the effect of human interventions represented by the worse repairs. The idea is that a worse repair increases 
the rate of defectives to a certain extent $(\beta+\Delta)$ for the following reasons: 1$)$ the faulty component is only partially repaired 2) human errors cause further damage, etc., as suggested by Pham and Wang (1996). The combination of the aging process and the worse repairs defines a deterioration cycle: aging-repairs-aging-repairs, etc., as illustrated in Figure 2.

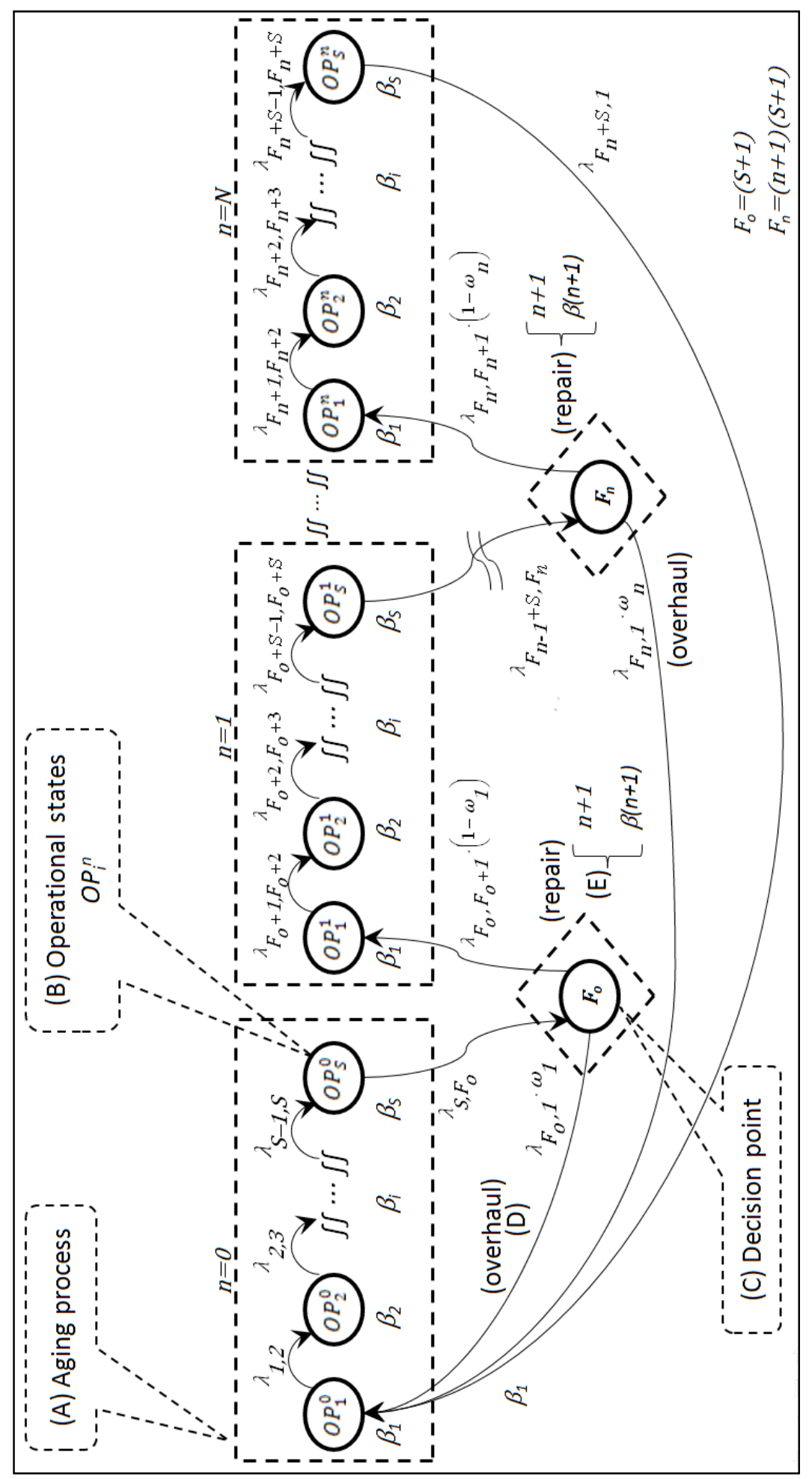


In the transition diagram, $S$ denotes the number of operational states of the aging process for any number of repairs n, and we used the index $F_{n}=(n+1)(S+1)$, to identify correctly the transitions between the different states. We can now define the dynamics of the production system as given by a hybrid state consisting of discrete and continuous components. The discrete components are denoted by $\xi(t)$ and $n(t)$, corresponding to the different states of the system at time $t$ and the number of worse repairs to date, respectively. Thus, the mode of the machine at time $t$ is given by $\xi(t) \in \Omega=\{1,2, \ldots, N(S+1\}$ such that:

$$
\xi(t)=\left\{\begin{array}{cc}
1 & \text { operational state } O P_{1}^{0} \text { at no failure } \\
2 & \text { operational state } O P_{2}^{0} \text { at no failure } \\
\vdots & \vdots \\
S & \text { operational state } O P_{S}^{0} \text { at no failure } \\
F_{O} & \text { the machine is at the } 1^{\text {st }} \text { failure } \\
1 & \text { operational state } O P_{1}^{1} \text { at the } 1^{\text {st }} \text { failure } \\
\vdots & \vdots \\
F_{n} & \text { the machine is at the } \mathrm{n}^{\text {th }} \text { failure } \\
1 & \text { operational state } O P_{1}^{n} \text { at the } \mathrm{n}^{\text {th }} \text { failure } \\
2 & \text { operational state } O P_{2}^{n} \text { at the } \mathrm{n}^{\text {th }} \text { failure } \\
\vdots & \vdots \\
S & \text { operational state } O P_{S}^{n} \text { at the } \mathrm{n}^{\text {th }} \text { failure }
\end{array}\right.
$$

The machine may randomly be at any of the proposed modes over an infinite horizon. The failure/repair/overhaul process is described using the semi-Markov Chain $\xi(t)$ characterized by transition rates $\lambda_{\alpha \alpha^{\prime}}(\cdot), \alpha, \alpha^{\prime} \in \Omega$, where the transitions $\lambda_{\alpha \alpha^{\prime}}$ from modes $\alpha$ to $\alpha^{\prime}$ satisfy the following conditions:

$$
\begin{aligned}
& P\left[\xi(t+\delta t)=\alpha \mid \xi(t)=\alpha^{\prime}, x(t)=x, n(t)=n\right] \\
= & \left\{\begin{array}{cl}
\lambda_{\alpha \alpha^{\prime}}(\cdot) \delta t+o(x, n, \delta t) & \text { if } \alpha \neq \alpha^{\prime} \\
1+\lambda_{\alpha \alpha^{\prime}}(\cdot) \delta t+o(x, n, \delta t) & \text { if } \alpha=\alpha^{\prime}
\end{array}\right.
\end{aligned}
$$

with:

$\lambda_{\alpha \alpha^{\prime}}(\cdot) \geq 0, \quad \lambda_{\alpha \alpha^{\prime}}(\cdot)=-\sum_{\alpha \neq \alpha^{\prime}} \lambda_{\alpha \alpha^{\prime}}(\cdot), \quad \forall \alpha, \alpha^{\prime} \in M \quad$ and $\lim _{\delta t \rightarrow 0} \frac{o(x, n, \delta t)}{\delta t}=\mathbf{0}$

The previous equation (2) denote the transition matrix $Q(\cdot)=\left\{\lambda_{\alpha \alpha^{\prime}}(\cdot)\right\}$ of the semi-Markov chain $\xi(t)$. We can improve the performance of the manufacturing system during its life cycle; with the use of the decision variable $\omega(\cdot)$; this variable controls the transition rate to the overhaul or to the worse repair. Hence, the matrix $Q(\cdot)$ depends on the decision variable $\omega(\cdot)$ and is defined by: 


$$
Q(\omega)=\left[\begin{array}{ccccccc}
\lambda_{1,1} & \lambda_{1,2} & 0 & 0 & 0 & \ldots & 0 \\
\ldots & \ldots & \ldots & \ldots & \ldots & \ldots & \ldots \\
\lambda_{F_{n}, 1} \cdot \omega & 0 & 0 & \lambda_{F_{n}, F_{n}} & \lambda_{F_{n}, F_{n}+1} \cdot(1-\omega) & \ldots & 0 \\
\ldots & \ldots & \ldots & \ldots & \ldots & \ldots & \ldots \\
\lambda_{N(S+1), 1} & 0 & 0 & 0 & 0 & \ldots & \lambda_{N(S+1), N(S+1)}
\end{array}\right]
$$

where $N$ represents the maximum number of failures during which the system remains operational. In the next section, we provide a detailed discussion about this parameter. At the operational state $O P_{i}^{n}$, we assume that the capacity constraint of the manufacturing system is defined by the control variable $u(\cdot)$ as follows:

$$
0 \leq u(\cdot) \leq U_{\max }
$$

where $U_{\max }$ is the maximum production capacity and $u(\cdot)$ is the production rate for the operational state $O P_{i}^{n}$. The control variables of the model are the production rate $u(\cdot)$ and the repair/overhaul switching strategy $\omega(\cdot)$. Thus, the set of admissible strategies $\Gamma(\cdot)$ that defines the feasible plan $(u(\mathrm{n}, \cdot), \omega(\mathrm{n}, \cdot))$ depends on the stochastic process $\xi(t)$, and is given by the following expression:

$$
\Gamma(\alpha)=\left\{(u(\mathrm{n}, \cdot), \omega(\mathrm{n}, \cdot)) \in R^{2}, \quad 0 \leq u(\mathrm{n}, \cdot) \leq u_{\max }, \quad 0 \leq \omega(\mathrm{n}, \cdot) \leq 1\right\}
$$

We next turn our attention to the continuous component of the hybrid state defined by the variable $x(t)$, which represents the inventory-backlog of parts produced. Since we consider that quality deterioration has the effect of increasing the rate of defectives, it is necessary to increment the demand rate to ensure that the production system satisfies the demand with flawless products. This condition leads to propose that the system dynamics evolves according to the following differential equation:

$$
\dot{x}(t)=u(t)-d[1+\beta(\mathrm{i}, \mathrm{n})], x(0)=x_{0}
$$

where $x_{0}$ is the given initial inventory level, $d$ denotes the demand rate and $\beta(\mathrm{i}, \mathrm{n})$ represent the function for the rate of defectives, $i$ describes the stage of the aging process and $n$ is the current number of repairs. Let us define $g(\cdot)$ as the running cost in state $\alpha \in \Omega$, when the current stock level is $x$ and the machine has already had its $n^{\text {th }}$ repair, then the cost function is defined by the following equation:

$$
\begin{aligned}
& g(\alpha, x, n, u, \omega)= \\
& =c^{+} x^{+}+c^{-} x^{-}+c^{\alpha} \\
& x^{+}=\max (0, x) \\
& x^{-}=\max (-x, 0) \\
& c^{\alpha}=c_{r} \cdot \lambda_{F_{n}, F_{n}+1} \cdot(1-\omega) \cdot \operatorname{Ind}\left\{\xi(t)=F_{n}\right\}+c_{o} \cdot \lambda_{F_{n}, 1} \cdot \omega \cdot \operatorname{Ind}\left\{\xi(t)=F_{n}\right\} \\
& \operatorname{Ind}\{\theta(t)\}=\left\{\begin{array}{l}
1 \text { if } \theta(t) \text { is true } \\
0 \text { otherwise }
\end{array}\right.
\end{aligned}
$$


where the constants $c^{+}$and $c^{-}$are used to penalize the inventory and backlog of parts, respectively; $c_{r}$ is the repair cost, and $c_{o}$ is the overhaul cost. In addition, the objective functional given by the expected discounted cost is:

$J(\alpha, x, n, u, \omega)=$

$$
=E\left\{\int_{0}^{\infty} e^{-\rho t} g(\cdot) d t \mid \alpha(0)=\alpha, x(0)=x, n(0)=n\right\}
$$

where $\rho$ denotes the discounted rate of the incurred cost. From what has been presented, it is apparent that the problem lies in determining an optimal control policy $\left(u^{*}(\cdot), \omega^{*}(\cdot)\right)$ where we seek to minimize the integral of the discounted cost $J(\cdot)$. Optimal policies are obtained from the value function defined as follows:

$$
\begin{aligned}
& v(\alpha, x, n)= \\
& \inf _{(u(\mathrm{n} \cdot), \omega(\mathrm{n} \cdot)) \in \Gamma(\alpha)} J(\alpha, x, n, u, \omega), \quad \forall \alpha \in \Omega, \quad x \in R, \quad n \in N
\end{aligned}
$$

The value function $v(\alpha, x, n)$ denotes the optimum value (in this case the minimum) of the integral of the discounted cost (9) and it satisfies specific properties called optimality conditions. In this respect regarding the principle of optimality, generally if $v(\cdot, t)$ denotes a cost-to-go function and if the initial time of the problem is $t=0$, then the change in the optimal function is made up of two parts: the incremental change of the discounted cost in the time interval $[0, t]$, and the change in the interval $[t, \infty]$. Then we can break up the minimization as follows:

$$
\begin{aligned}
& v(\alpha(t), x(t), n(t), t)= \\
& \inf _{\substack{u(s), \omega(s) \\
0 \leq s \leq \infty}} E\left\{\begin{array}{c|c|}
\int_{0}^{t} e^{-\rho t} g[(\alpha(s), x(s), n(s), u(s), \omega(s)] d s \\
+
\end{array} \mid \alpha(t), x(t), n(t)\right\}
\end{aligned}
$$

The control actions should be chosen to minimize the sum of these two terms. Additionally, the derivation of the optimality conditions must take into account the randomness of $\alpha$ (this explain the expectation operation), and the discounted rate $\rho$. We can then apply the conditional expectation operation $\widetilde{E}$ (i.e., for any function $H(\alpha)$, $\widetilde{E} H(\alpha(t+\delta t))=E\{H(\alpha(t+\delta t)) \mid \alpha(t)\})$ and if we perturb $t$, for any $\delta t$, equation (11) can be approximated by:

$$
\begin{aligned}
v(\alpha(t), x(t), n(t), t)= & \inf _{\substack{u(s), \omega(s) \\
t \leq s \leq t+\delta t}} \tilde{E}
\end{aligned}\left\{\begin{array}{c}
g[(\alpha(t), x(t), n(t), u(t), \omega(t)] \delta t \\
+ \\
\frac{1}{1+\rho \delta t} v[\alpha(t+\delta t), x(t+\delta t), n(t+\delta t), t+\delta t]
\end{array}\right\}+o(\delta t)
$$


Assuming that $v(\cdot)$ is differentiable, we can expand its derivative, and the expectation operation $\tilde{E}$. Then for small $\delta t$ and after some manipulations equations (12) becomes:

$$
\begin{aligned}
& \rho v(\alpha(t), x(t), n(t), t)= \\
& \inf _{u(t), \omega(t)}\left\{\begin{array}{c}
g[(\alpha(t), x(t), n(t), u(t), \omega(t)] \delta t+ \\
\frac{\partial v}{\partial x}[\alpha(t), x(t), n(t), t] \delta x(t)+\frac{\partial v}{\partial t}[\alpha(t), x(t), n(t), t] \delta t \\
+\sum_{\alpha \prime} v\left[\alpha^{\prime}, x(t), n(t), t\right] \lambda_{\alpha^{\prime} \alpha(\mathrm{t})} \delta t
\end{array}\right\}+o(\delta t)
\end{aligned}
$$

We have eliminated the expectation symbol with the summation term. If we replace $\delta x(t)$ by $\delta x(t)=\dot{x}(t) \delta t$ and do other manipulations, we get:

$$
\begin{aligned}
\rho v(\alpha, x, n, t) & -\frac{\partial v}{\partial t}[\alpha, x, n, t]= \\
& \inf _{u(t), \omega(t)}\left\{g[\alpha, x, n, u, \omega]+\frac{\partial v}{\partial x}[\alpha, x, n, t] \dot{x}+\sum_{\alpha^{\prime}} v\left[\alpha^{\prime}, x, n, t\right] \lambda_{\alpha^{\prime} \alpha}\right\}
\end{aligned}
$$

We observe that none of the functions $g(\cdot)$ and $\dot{x}(\cdot)$ are functions of $t$ explicitly. Furthermore since the time horizon is infinite and a steady state distribution exists for $\alpha$, equations (14) is independent of $t$. Based on this and replacing the summation term by the generator $\mathrm{Q}(\cdot)=\left\{\lambda_{\alpha \alpha^{\prime}}(\cdot)\right\}$, equations (14) can be further simplified to:

$$
\begin{aligned}
& \rho v(\alpha, x, n)= \\
& \min _{(u, \omega) \in \Gamma(\alpha)}\left\{g[\alpha, x, n, u, \omega]+\frac{\partial v}{\partial x}[\alpha, x, n] \dot{x}+Q(\cdot) v[\alpha, x, \varphi(\xi, n)](\alpha)\right\}
\end{aligned}
$$

These are the fundamental equations called Hamilton-Jacobi-Bellman (HJB) equations that we use to determine the optimal control policy. Further details about how the HJB equations are obtained can be consulted in Rishel (1975) and Gershwin (2002). In order to complete our formulation, since the overhaul restores the rate of defectives $\beta$ to initial conditions, and that the effect of worse repairs is to increase this rate, we define, at a jump time $\tau$ for the process $\xi(t)$, a reset function $\varphi(\xi, n)$. This reset function describes any discontinuity that may occur at a jump time $\tau$ in the modes of the manufacturing system and is defined as follows:

$$
\varphi(\xi, n)=\left\{\begin{array}{c}
n+1 \text { if } \xi\left(\tau^{+}\right)=O P_{1}^{n} \quad \text { and } \xi\left(\tau^{-}\right)=F_{n} \\
0 \quad \text { if } \xi\left(\tau^{+}\right)=O P_{1}^{0} \text { and } \xi\left(\tau^{-}\right)=F_{n} \\
n \quad \text { otherwise }
\end{array}\right.
$$

It remains to determine the optimal policy $\left(u^{*}, \omega^{*}\right)$, when the value function is available, an optimal control policy can be obtained, as presented in the HJB equations. However, the issue is that it is extremely complicated to solve them analytically, because they lead to intractable problems. Also it remains to be specified how exactly the function $\beta(\mathrm{i}, \mathrm{n})$ will model the quality deterioration phenomenon. More details about these concerns are provided in the next sections. 


\subsection{Deteriorating systems}

The object of this section is to detail the expressions that deal with the deterioration phenomenon. In this respect we find that some authors relate the deterioration of the machine with the quality of the parts produced. For example Kim and Gershwin (2008), proposed a general model that represents the wear of the machine and that also indicates different quality levels, implying a deterioration of quality. In the same direction Colledani and Tolio (2011) suggest that the degrading process of the machine has a continuous deterioration on the parts' quality. Even in the area of quality it exists a control charts for trends, where a gradual change in the part's feature is expected and considered to be normal given for instance by tool wears, as indicated in Besterfield (2009). Therefore in our paper we conjecture a relation between deterioration and quality that increases the rate of defectives $\beta$ by the combination effect of two factors (aging process and human interventions), as denoted by the following expression:

$$
\beta(i, n)=\zeta(n) \cdot[1+\mathrm{p}(i)]
$$

where $i$ is the current stage of the aging process and satisfies $1 \leq i \leq S$. Additionally $n$ is the current number of repairs, the function $\zeta(n)$ models the effect of worse repairs on the rate of defectives for a given $n$, and $\mathrm{p}(i)$ represents the effect of the aging process for the current $i$. To detail how expression (17) works, we concentrate first on the effect of the aging process $\mathrm{p}(i)$. The idea is that the wear of the production system implies certain changes, thereby we model the aging process with a set of discrete operational states $O P_{i}^{n}$ with an increasing rate of defectives $\left(\beta_{0}<\beta_{1}<\cdots<\beta_{\mathrm{N}}\right)$ as indicated by Kim (2005), where the movement from state to state represents the deterioration of quality. Thus the increment in the rate of detectives for every operational state $O P_{i}^{n}$ is given by the following expression:

$$
p(i)=b_{b}^{(i-1)} \cdot d_{b}
$$

where $\mathrm{p}(i)$ represents the percentage that will increment the rate of defectives at a given stage $i$ of the aging process, $d_{b}$ is a constant with a value near to zero, and $b_{b}$ denotes the common ratio of the expression. The constant $b_{b}$ is useful for adjusting the expression (18) to other types of machines. Function (18) says that the increment $\Delta_{i}$ in the rate of defectives for every operational state $O P_{i}^{n}$ is not fixed; it increases, as presented in Figure 3a. The increment $\Delta_{i}$ can be defined from expression (18) as follows:

$$
\Delta_{i}=\mathrm{p}(i+1)-\mathrm{p}(i)
$$

The quality deterioration modeling of equation (17) is complemented with the effect of human interventions, defined by the current number of worse repairs. Several authors have successfully related the number of repairs with the deterioration of the system as in Leung (2001) and Lam (2007). Moreover they have observed a certain trend in the deterioration, and even their results have been valid in real industrial data as reported in Lam and Chan (1998). Consequently given the relations between repairs-deterioration and deterioration-quality, we propose an increasing function $\zeta(n)$, where the rate of defectives increases gradually as the number of worse repairs grows, according to the following expression: 


$$
\zeta(n)=\frac{d_{b}}{a_{b}^{(n-1)}}
$$

where $d_{b}$ is the value of the rate of defectives at initial conditions, $n$ denotes the current number of worse repairs at time $t$, and $a_{b}$ describes the common ratio of the expression. The advantage of equation (20) is that we can adjust the value of the parameter $a_{b}$ to modify the trend of the rate of defectives for a specific type of machine. Figure $3 \mathrm{~b}$ presents the graph for this expression (20), where the increment $\Delta_{n}$ can be obtained as follows:

$$
\Delta_{n}=\zeta(n+1)-\zeta(n)
$$

We claim in Figure $3 b$ that after a worse repair the rate of defectives grows a certain amount $\Delta_{n}$, and due to the accumulated wearing of the machine, this increment $\Delta_{n}$ is not constant; it has a low value for the first repairs and it follows an increasing trajectory as suggested in Lam et al. (2004).

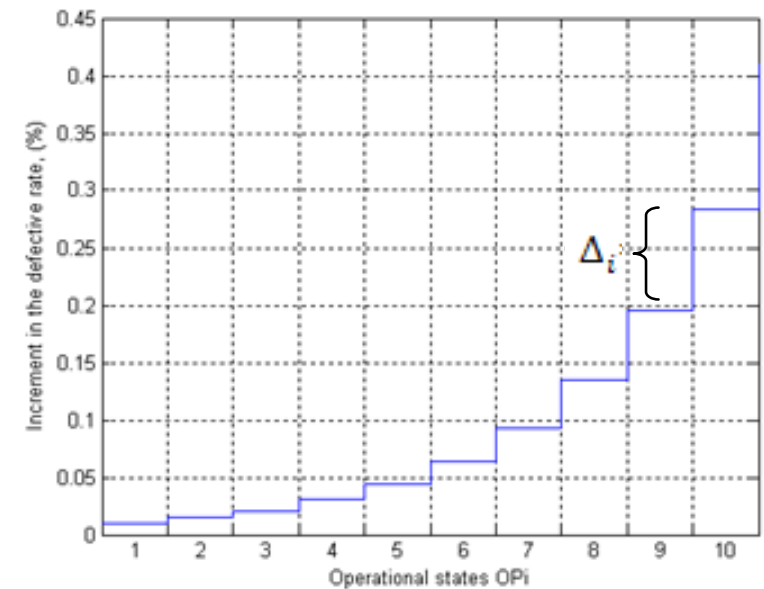

(a) caused by the aging process

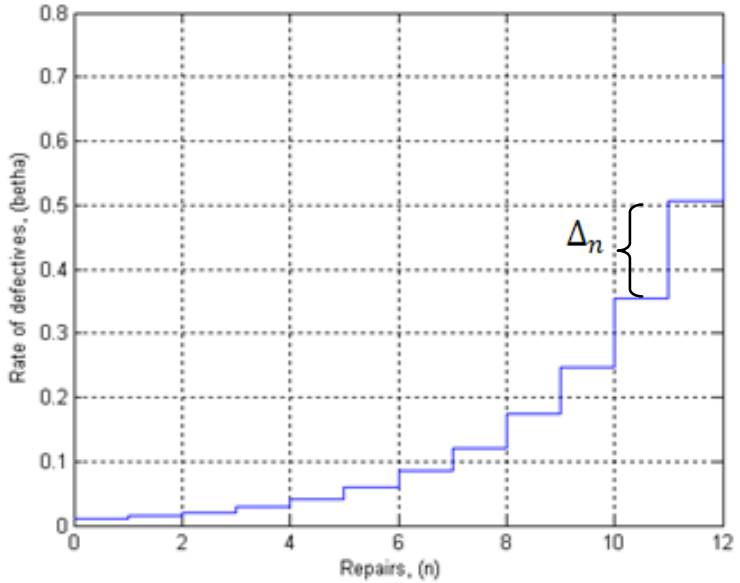

(b) caused by the worse repairs

Figure 3: Trend of the quality deterioration

By incorporating expression (18) and $(20)$ into $\beta(i, n)$, finally it derives in:

$$
\beta(i, n)=\frac{d_{b}}{a_{b}^{(n-1)}} \cdot\left[1+b_{b}^{(i-1)} \cdot d_{b}\right]
$$

We conclude this section with the following remark. The consideration of the deterioration of quality yields to a new model, where the quality of the parts produced is negatively affected. In essence equation (22) says that the quality deterioration has two components; the natural wear of the machine given by the set of operational states and the effect of the worse repairs. These two components summarize the effect of other possible presented factors. Because of these innovative characteristic, our formulation leads to a semi-Markov model since the information that is available to the decision maker at each instant of time $t$, includes the state of the production system, denoted by the stock level, as well as the machine's history, with the number of repairs and the set of operational states. 


\subsection{Numerical approach}

Notwithstanding the difficulty in solving the HJB equation (15), fortunately it is possible to obtain an approximation of the control policy through the application of a numerical method conceived for optimal control models. This numerical method is based on the Kushner approach, and the crux of this technique consists in approximating the continuous value function $v(\alpha, x, n)$ by the discrete function $v^{h}(\alpha, x, n)$ and the gradient $\frac{\partial v}{\partial x}(\alpha, x, n)$ by the following expression:

$$
\begin{aligned}
& \frac{\partial v}{\partial x}(\alpha, x, n)= \\
& \begin{cases}\frac{1}{h}\left(v ^ { h } \left((\alpha, x+h, n)-v^{h}((\alpha, x, n)\right.\right. & \text { if } \quad \dot{x} \geq 0 \\
\frac{1}{h}\left(v ^ { h } \left((\alpha, x, n)-v^{h}((\alpha, x-h, n)\right.\right. & \text { if } \quad \dot{x}<0\end{cases}
\end{aligned}
$$

where $h$ is a discrete increment associated with the state variable $x$. More details about the Kushner approach can be seen in Kushner and Dupuis (1992). The application of the numerical method implies the use of a discrete equation for every mode of the machine that in this case it is defined as follows:

$$
\begin{aligned}
& v^{h}(\alpha, x, n)= \\
& \min _{(u(\mathrm{n}, \cdot), \omega(\mathrm{n}, \cdot)) \in \Gamma(\alpha)}\left[( \rho + | \mathrm { q } _ { \alpha \alpha } | + \frac { | r | } { \mathrm { h } } ) ^ { - 1 } \left(g(\cdot)+v^{h}(\alpha, x+h, n) \frac{|r|}{\mathrm{h}} \operatorname{Ind}\{r \geq 0\}\right.\right. \\
& \left.\left.+v^{h}(\alpha, x-h, n) \frac{|r|}{\mathrm{h}} \operatorname{Ind}\{r<0\}+Q(\cdot) v(\alpha, x, \varphi(\mathrm{n}, \xi))(\alpha)\right)\right]
\end{aligned}
$$

where $r=u(\cdot)-d[1+\beta(\mathrm{i}, \mathrm{n})]$. In general terms, the numerical method reduces the complexity of the original continuous control problem defined by the HJB equation (15) to a discrete semi-Markov decision process (24) with finite state space and finite action space. The technical advantage of the numerical method is that the discrete semi-Markov process (24) is much easier to solve than the continuous version. Moreover, we can interpret its coefficients as the transitions probabilities between the different points defined in the computational domain $G_{x n}^{h}$. Eventually, the obtained discrete semi-Markov process (24) can be solved by the policy improvement technique or value iteration methods.

\section{Numerical example}

This section provides a numerical example of the manufacturing system presented in section 2. The computation algorithm applied to solve the discrete semi-Markov process (24) is based on the policy improvement technique. Clearly, the solution $v^{h}(\alpha, x, n)$ of equation (24) is a discrete approximation that converges to the continuous function $v(\alpha, x, n)$ of equation (10). The algorithm that leads us to the optimal policy requires that 
we follow a specific sequence of steps, which can be consulted in detail in Kushner and Dupuis (1992) and Kenne et al. (2003). Without loss of generality, we consider three operational states for the stages of the aging process; in other words, this means that $S=3$. The numerical method is then applied with the data presented in Table 1, where the value of each required parameter is defined.

\begin{tabular}{lccccc}
\hline Parameter: & $\mathrm{u}_{\max }$ (units/hr) & $\mathrm{d}$ (units/hr) & $\mathrm{h}$ & $\rho$ & $\mathrm{c}+(\$ / \mathrm{units} / \mathrm{hr})$ \\
Value: & 5 & 3 & 0.5 & 0.9 & 1 \\
\hline Parameter: & $\mathrm{c}-(\$ / \mathrm{units} / \mathrm{hr})$ & $\mathrm{c}_{\mathrm{r}}(\$)$ & $\mathrm{c}_{\mathrm{o}}(\$)$ & $\mathrm{N}$ & $\mathrm{a}_{\mathrm{b}}$ \\
Value: & 200 & 20 & 660 & 20 & 0.815 \\
\hline Parameter: & $\mathrm{b}_{\mathrm{b}}$ & $\mathrm{d}_{\mathrm{b}}$ & $\lambda_{12}(1 / \mathrm{hr})$ & $\lambda_{23}(1 / \mathrm{hr})$ & $\lambda_{34}(1 / \mathrm{hr})$ \\
Value: & 3.5 & 0.01 & 0.5 & 0.2 & 0.1 \\
\hline Parameter: & $\lambda_{\left(F_{n}+1\right),\left(F_{n}+2\right)}$ & $\lambda_{\left(F_{n}+2\right),\left(F_{n}+3\right)}$ & $\lambda_{\left(F_{n-1}+S\right), F_{n}}$ & $\lambda_{F_{n}, 1}$ & $\lambda_{F_{n},\left(F_{n}+1\right)}$ \\
& $(1 / \mathrm{hr})$ & $(1 / \mathrm{hr})$ & $(1 / \mathrm{hr})$ & $(1 / \mathrm{hr})$ & $(1 / \mathrm{hr})$ \\
Value: & 0.5 & 0.2 & 0.1 & 0.02 & 2 \\
\hline Parameter: & $\omega_{\min }$ & $\omega_{\max }$ & & & \\
Value: & 0 & 1 & & & \\
\hline
\end{tabular}

Table 1. Parameters of the numerical example

The implementation of the numerical technique also requires the use of a discrete grid for the inventory level $x$ and the number of repairs $n$. The grid is denoted by $G_{x n}^{h}$ and defines the computational domain as follows:

$$
G_{x n}^{h}=\{(\mathrm{x}, \mathrm{n}):-10 \leq \mathrm{x} \leq 30, \quad 0 \leq \mathrm{n} \leq 20, \quad \mathrm{~h}=0.2\}
$$

The manufacturing system will be in conditions to satisfy the demand rate $d$ over an infinite horizon and reach steady state only if the system is feasible. This implies the satisfaction of the following condition:

$$
u_{\max } \cdot \pi_{1}+\ldots+u_{\max } \cdot \pi_{3 \mathrm{~N}} \geq d \cdot[1+\beta(i, n)]
$$

where $\pi_{1}, \ldots, \pi_{3 N}$ are the limiting probabilities for the operational modes of the machine, which are normally computed as follows:

$$
\pi_{i} \cdot Q(\cdot)=0 \quad \text { and } \quad \sum_{i=1}^{N(S+1)} \pi_{i}=1
$$

It is pertinent to note that the feasibility condition (26) is satisfied by the selected values of the parameters presented in Table 1 . With the values of $\mathrm{a}_{b}$ and $\mathrm{d}_{b}$ applied in expression (22), it follows that the limit of feasibility is reached at repair $N=20$. This implies that the semi-Markov generator $Q(\cdot)$ is composed of 60 operational states $O P_{i}^{n}$ and 20 failure states $F_{\mathrm{n}}$. The results presented in Figures 4 to 8 were obtained with the data presented in Table 1, and they illustrate the optimal control policies for the production and repair/overhaul switching strategy. 


\subsection{Production policy}

The optimal production policy $u^{*}(\alpha, x, n)$ is illustrated in Figure 4, and indicates the production rate of the manufacturing systems applied in the operational states $O P_{3}^{n}$ for any number of repairs $n$ and stock level $x$. From Figure 4, it follows that the production policy divides the plan $(x, n)$ into three regions in which the production rate is set to $u_{\max }, d$, and 0 , respectively. Moreover, we observe that the trend in the production thresholds is a clear indicator of the effect of the quality deterioration. It turns out that the more the number of repairs increases, the more the optimal stock level increases as well.

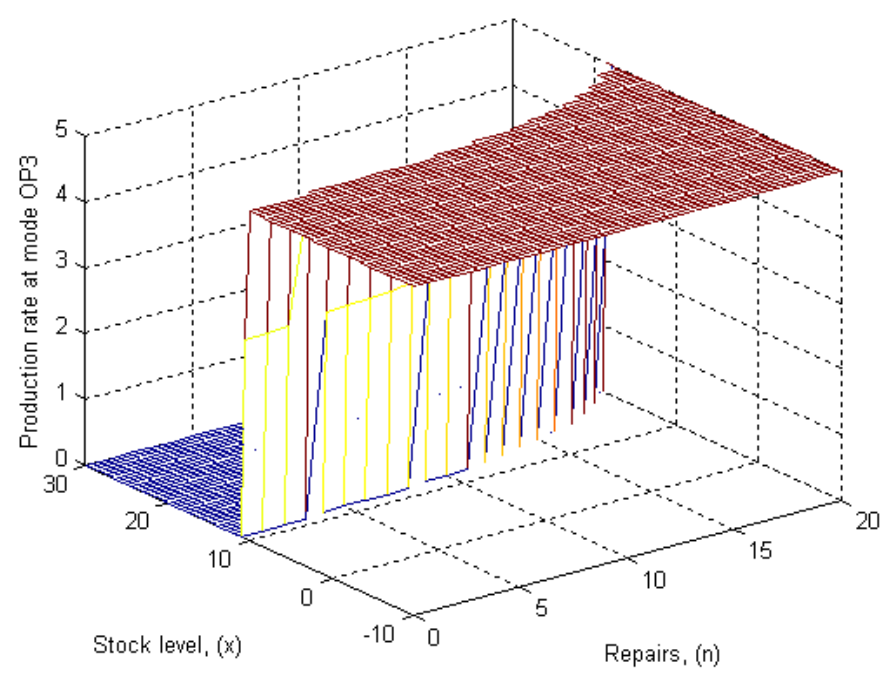

Figure 4: Production rate of the manufacturing system for the operational states $O P_{3}^{n}$

To better illustrate the optimal production policy, we use its boundary presented in Figure 5a, where we identify the optimal stock level $Z_{p i}^{*}(\cdot)$ applied in three stages of the operational states. In Figure 5a, we observe that $Z_{p 1}^{*}(\cdot)<Z_{p 2}^{*}(\cdot)<Z_{p 3}^{*}(\cdot)$; this is given because as the machine moves in the operational states, the amount of product to hedge against a breakdown increases. An idealistic case is when the machine has high availability; for instance, $99 \%$. This would imply a general reduction in the production thresholds for all the operational states, as presented in Figure 5b. In this figure, the failure takes so long to occur that the production thresholds $Z_{p 1}^{*}(\cdot)$ and $Z_{p 2}^{*}(\cdot)$ are equal to zero and even $Z_{p 3}^{*}(\cdot)$ is lower, compared with Figure 5a. We note that when the production system has lower availability, it is necessary to maintain a certain amount of inventory in the operational states, as presented in Figure 5a, where according to the data of Table 1, the system has an availability of $97 \%$. 


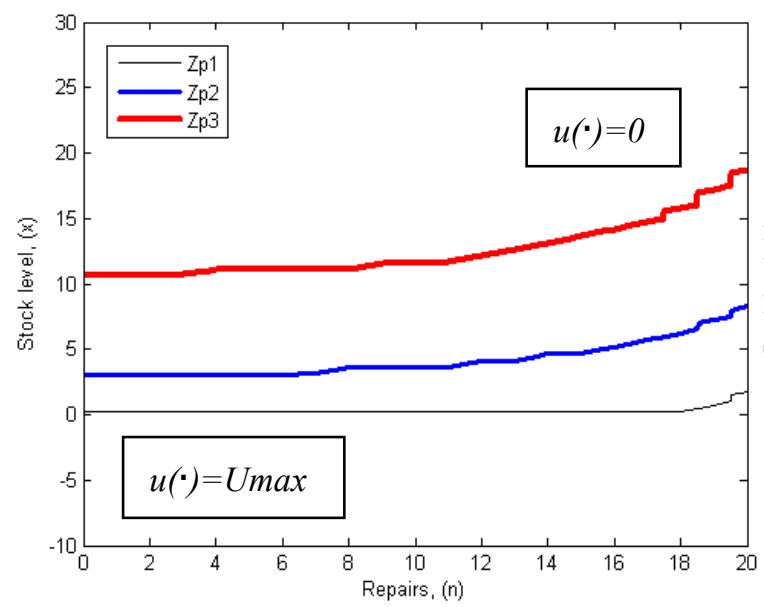

(a) With data of Table 1

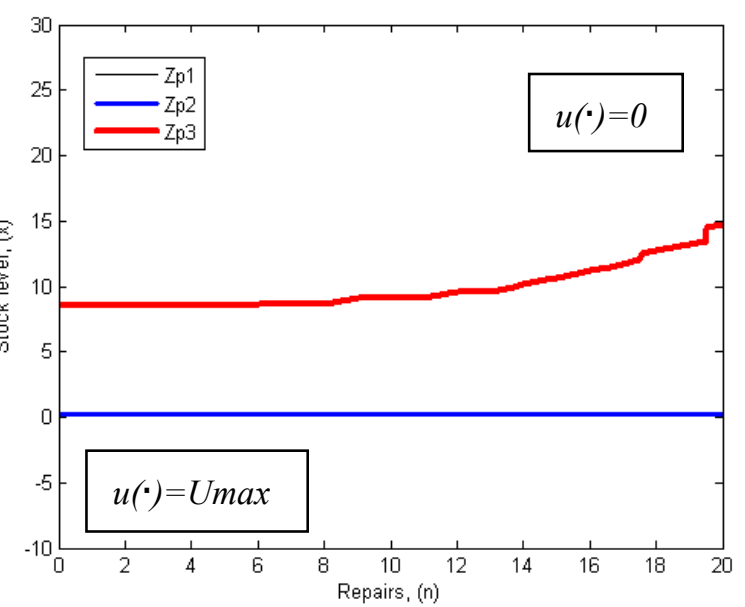

(b) With high availability data

Figure 5: Production trace $Z_{p i}^{*}(\cdot)$

It is remarkable that the trend in the optimal thresholds is influenced by the function applied to model the quality deterioration. This is apparent because the trend in the production thresholds presented in Figure 4 and Figure 5a are very similar to the graph of Figure 3. To summarize, the optimal production threshold can be defined using the switching trend presented in Figure 4 and Figure 5a, and the underlying pattern of this policy denotes a machine rate of defectives dependent hedging point policy, which for the numerical illustration, is given by the following equations:

$$
\begin{aligned}
& u(1,)^{*}=\left\{\begin{array}{cc}
u_{\max } & \text { if } x(t)<Z_{p 1}^{*}(\cdot) \\
d & x(t)=Z_{p 1}^{*}(\cdot) \\
0 & x(t)>Z_{p 1}^{*}(\cdot)
\end{array}\right. \\
& u(2, \cdot)^{*}=\left\{\begin{array}{cc}
u_{\max } & \text { if } x(t)<Z_{p 2}^{*}(\cdot) \\
d & x(t)=Z_{p 2}^{*}(\cdot) \\
0 & x(t)>Z_{p 2}^{*}(\cdot)
\end{array}\right. \\
& u(3,)^{*}=\left\{\begin{array}{cc}
u_{\max } & \text { if } x(t)<Z_{p 3}^{*}(\cdot) \\
d & x(t)=Z_{p 3}^{*}(\cdot) \\
0 & x(t)>Z_{p 3}^{*}(\cdot)
\end{array}\right.
\end{aligned}
$$

where $Z_{p i}^{*}(\cdot)$ is the function that gives the optimal production threshold for the operational states of the aging process, with the threshold level illustrated in Figure 5a. In general, it is observed that the production threshold increases as the machine moves in the operational states, and the following condition holds: $Z_{p 1}^{*}(\cdot) \leq Z_{p 2}^{*}(\cdot) \leq Z_{p 3}^{*}(\cdot)$. 


\subsection{Repair/overhaul switching policy}

The repair/overhaul switching policy obtained is presented in Figure 6, where we observe that the plan $(x, n)$ is divided into two regions. The decision variable $\omega$ is set to its maximum or minimum value, depending on the current stock level and the number of worse repairs. The maximum value, $\omega=1$, defines the most convenient time to conduct the overhaul, and conversely, the minimum value, $\omega=0$, suggests when to perform the worse repair. It is apparent that the role of the control variable $\omega$ is to synchronize the two available maintenance options.

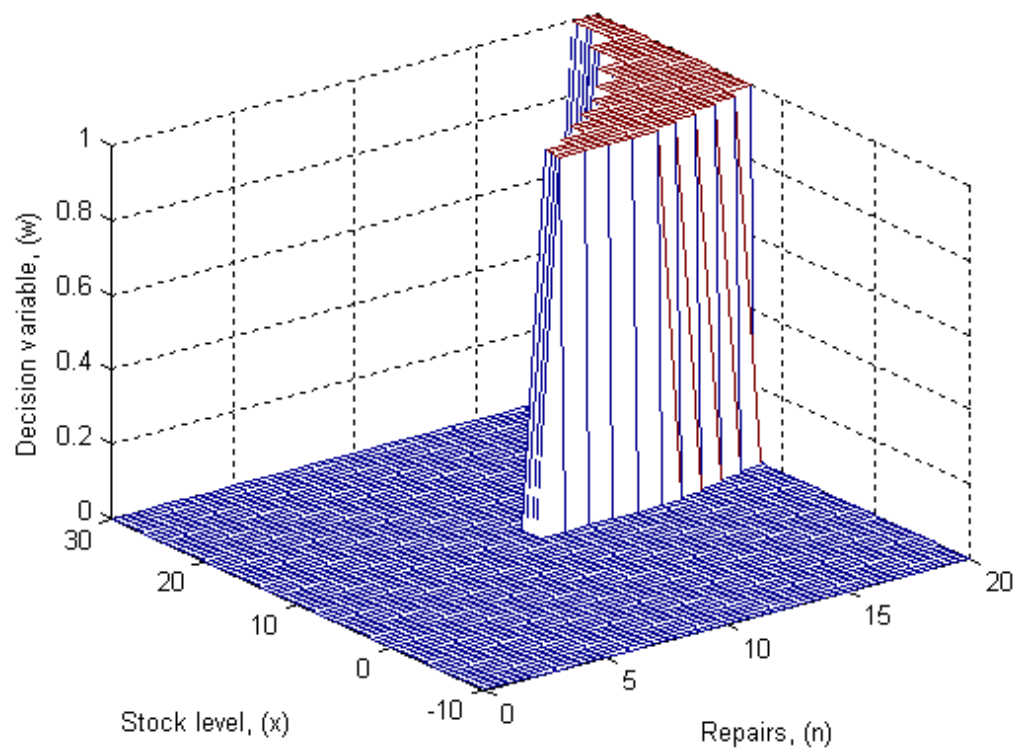

Figure 6: Repair/Overhaul policy

To facilitate the analysis of the repair/overhaul switching policy, its boundary denoted by $D_{n}(\cdot)$ is used. The boundary divides the computational domain $G_{x n}^{h}$ into two zones, according to the type of maintenance performed. The zones are presented in Figure 7, and are defined as follows:

- Zone $A$, here is justified the cost of an overhaul, and therefore this type of maintenance is recommended; furthermore, the decision variable $\omega$ is set to its maximum value.

- Zone $B$, in this zone, the overhaul activity is not recommended, but rather, the worse repair is the maintenance option suggested, and the variable $\omega$ is set to its minimum value.

By analyzing Figure 7, it seems logical not to perform the overhaul maintenance if the number of repairs is low, since this means that the level of deterioration is also low, and this condition does not justify the high cost of an overhaul. Moreover, we observe that the overhaul zone is located in the positive stock area, which leads to the observation that, much more time and resources are needed to perform an overhaul than to carry out worse repairs. Consequently, some amount of stock is required to circumvent the loss of demand fulfillment while the machine is out of operation, and an overhaul is being performed. 


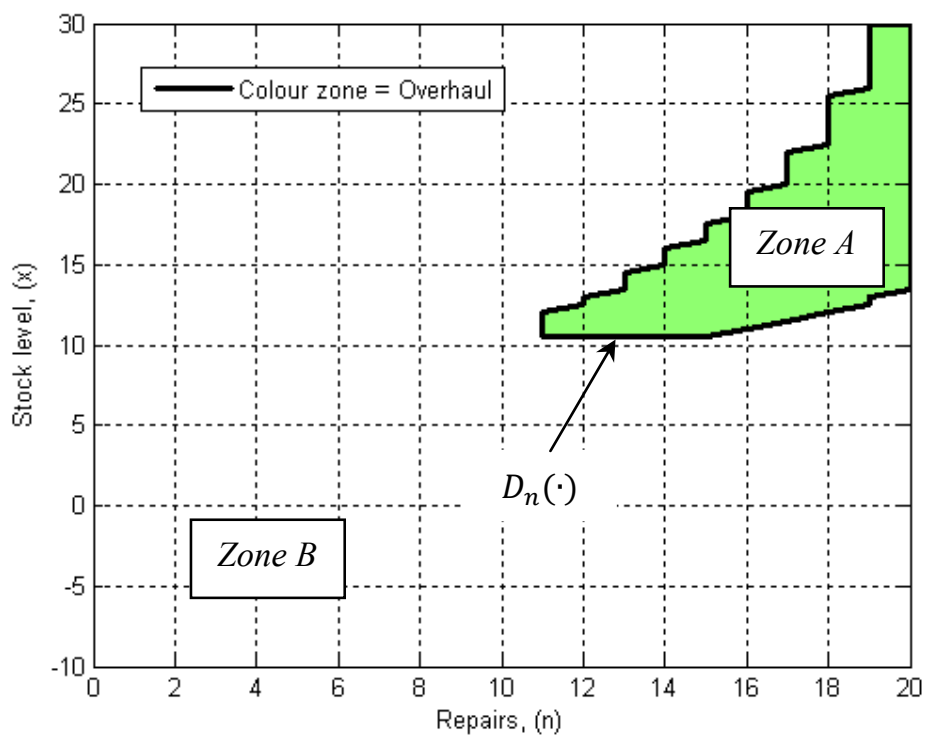

Figure 7: Trace of the repair/overhaul policy

In Figure 8, we present the intersection of the production and the repair/overhaul policy. As we can observe in this figure, when we include the boundary of the production policy, only a part of the overhaul zone is feasible since the stock level is limited by the production threshold $Z_{p}^{*}(\cdot)$. Even though the overhaul zone is bigger, we stress that the intersection of both policies defines the feasible zone $A^{\prime}$ to implement in the manufacturing system. Thus this condition must be considered in determining the repair/overhaul policy.

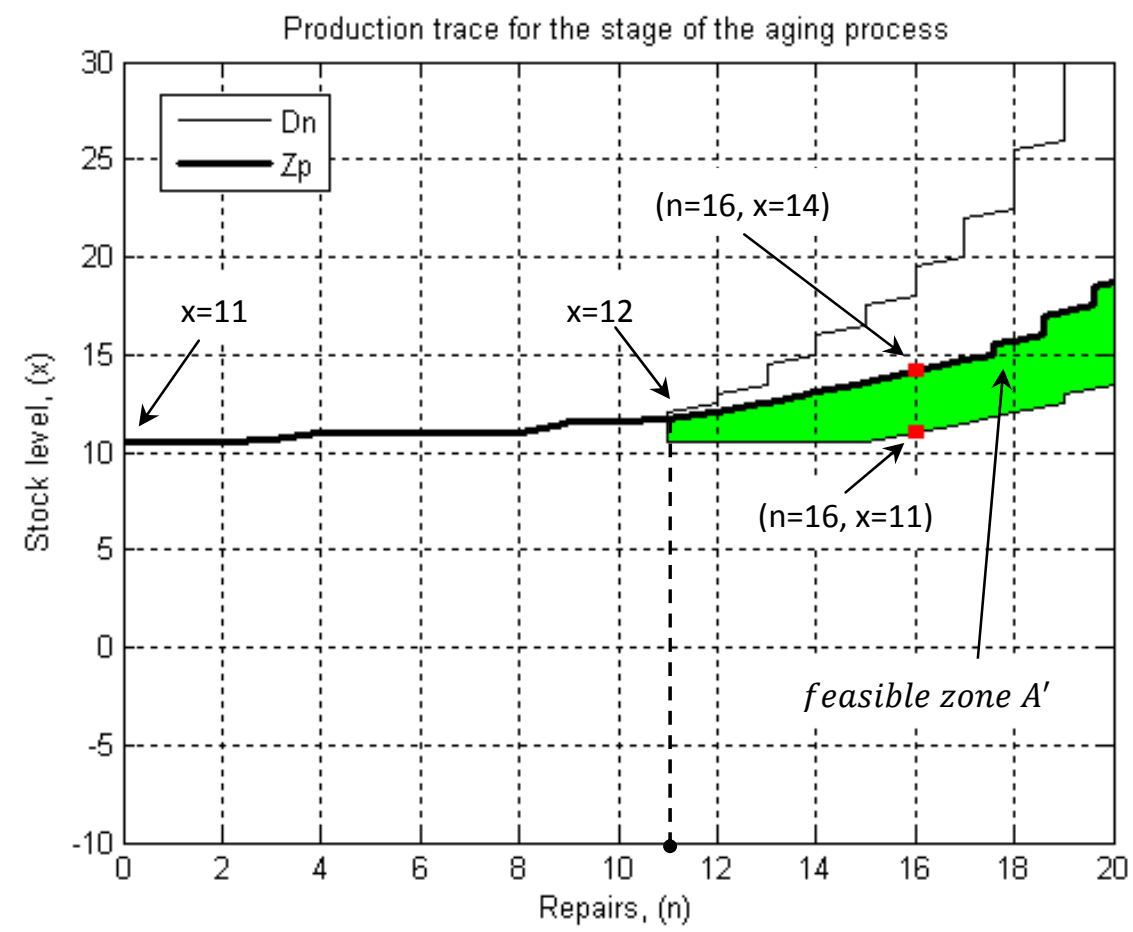

Figure 8: Intersection of the production and the repair/overhaul trace 
It should be pointed out that repair/overhaul activities are triggered according to the machine rate of defectives dependent policy as observed in Figure 8, and that this optimal policy has a bang-bang structure. Let $R_{n}(\cdot)$ denote a function with value 1 if an overhaul is undertaken after the $\mathrm{n}^{\text {th }}$ failure, and 0 if a repair is performed. Based on the obtained results and with such a notation, the repair/overhaul policy is given by the following expression:

$$
R_{n}(\cdot)^{*}=\left\{\begin{array}{cc}
1 & \text { if } s(\cdot) \in \text { zone } A^{\prime} \text { defined by } D_{n}(\cdot) \text { and } Z_{p i}^{*} \\
0 & \text { otherwise }
\end{array}\right.
$$

with

$$
s(\cdot)=(n(t), x(t))
$$

where the coordinate $s(\cdot)$ is located in the grid $G_{x n}^{h}$. From what has been presented, it is valid to say that the joint optimal production and repair/overhaul control policies are defined by the equations (28)-(32). Based on the obtained results, it is evident that the production and the repair/overhaul policies can be completely characterized with the control parameters $Z_{p i}(\cdot)$ and $D_{n}(\cdot)$.

\section{Model implementation}

Figure 5a and Figure 8 are used in the implementation of the obtained results. At initial time, the machine is in the operational state $O P_{1}^{0}$, and since it is a brand new machine, there is no need to maintain a stock of products, hence the production threshold is $Z_{p 1}^{*}(0)=0$ and the number of repairs is equal to zero. As soon as the machine is put in operation, its rate of defectives starts deteriorating. While awaiting the first failure, the machine will eventually move to the operational state $O P_{3}^{0}$ where the production threshold increases to $Z_{p 3}^{*}(0)=11$. When the first failure occurs, the machine is repaired. This means that the rate of defectives will increase, since the repair does not consider any reduction in the rate of defectives. With the first repair, the machine becomes operational once again, and enters the operational state $O P_{1}^{1}$, from this point, the production system will behave in a similar pattern until the number of repairs reaches $n=11$. At this point, the production threshold at the operational state $O P_{3}^{11}$ increases to $Z_{p 3}^{*}(11)=12$, because of the deterioration phenomenon, and also this point indicates that the overhaul is beginning to be carried out.

Figure 9 illustrates the implementation of the control policy when the number of repairs is $n=16$. Let us assume that the machine has already experienced its $16^{\text {th }}$ repair, and is waiting for its next failure in the operational state $O P_{3}^{16}$. Meanwhile, the failure does not occur, the production threshold is set to $Z_{p 3}^{*}(16)=14$. This value is used to determine the correct production rate. When the failure arrives, if the current stock level $x$ is inside the interval $(11,14)$ defined by $D_{n}$ and $Z_{p 3}^{*}(16)$, then the overhaul is conducted, otherwise the machine is repaired. The benefit of the overhaul is that it restores the machine to initial conditions. Conversely, with the repair, the machine will continue deteriorating since it will eventually experience another failure. In the numerical illustration, the maximum number of repairs that the machine can experience is 20 , as discussed in section 4 , after 
which it is restored to brand new condition, regardless of its state. Additionally, the rate of defectives following an overhaul is reduced to initial conditions.

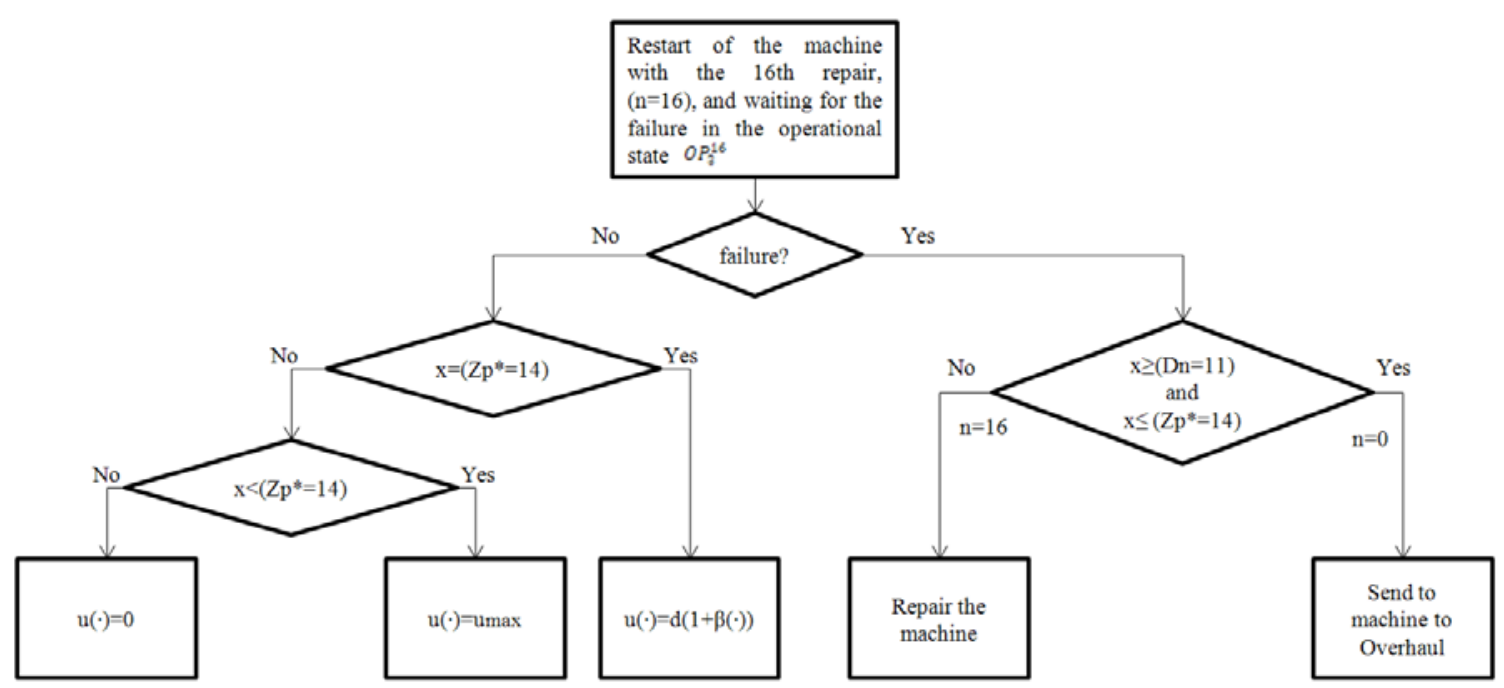

Figure 9: Model implementation diagram

To observe how these results are influenced by some of the parameters used in this model, in the next section we verify the structure of the obtained control policy. To that end, a sensitivity analysis is performed to ensure the consistency of the control policy and to illustrate the usefulness of the control approach.

\section{Sensitivity and results analysis}

In this section, we analyze different manufacturing scenarios involving changes in the cost parameters. The purpose is to illustrate the effect of cost variation on the optimal control policies and to determine if it is characterized consistently by the control factors $Z_{p i}(\cdot)$ and $D_{n}(\cdot)$. The sensitivity of the control policies is analyzed according to the variation of the cost of inventory, the cost of backlog, the cost of overhaul and the repair cost.

\subsection{Variation of the inventory cost}

We shall begin the sensitivity analysis with a discussion about the inventory cost. For this, we analyze the production threshold $Z_{p 3}^{*}(\cdot)$, for three different inventory cost values $c^{+}=1,3$ and 6 . We point out that this threshold $Z_{p 3}^{*}(\cdot)$ is applied in the operational states $O P_{3}^{n}$. From the results of Figure 10, it is observed that the more the inventory cost increases, the more the production threshold decreases, since we see that with a higher inventory cost, for instance $c^{+}=6$, the more the product stock is penalized, and with lower cost, such as $c^{+}=3$ and $c^{+}=1$, there is more liberty to maintain stock, and so the production threshold increases. Furthermore, the influence of quality deterioration on the optimal threshold is also evident because it increases as the number of repairs grows. 


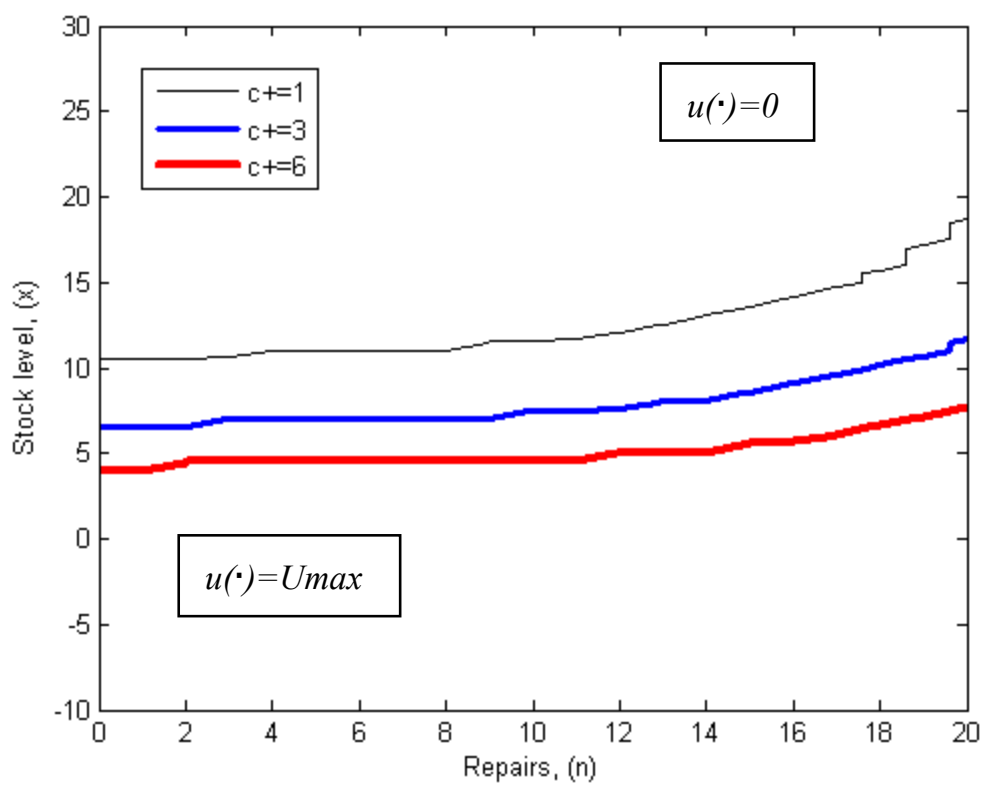

Figure 10: Variation of the inventory cost and its effect on the production threshold $Z_{p 3}^{*}(\cdot)$

As a matter of interest, in Figure 11, we present the effect of the variation of the inventory cost $c^{+}$on the repair/overhaul policy. We observe in this figure that when the inventory cost is low, for instance $c^{+}=1$, the overhaul zone $A^{\prime}$ is confined to a bigger area in the computational domain. From the repair/overhaul trace $D_{n}\left({ }^{\circ}\right)$, it is seen that the minimum number of repairs needed before considering the possibility of the overhaul is $n^{*}=10$. When the inventory cost increases to $c^{+}=1.5$, the overhaul zone $A^{\prime}$ reduces, adjusting the number of repairs to $n^{*}=13$. This condition is explained by the fact that one of the parameters involved in the determination of the overhaul zone $A^{\prime}$ is the inventory cost. In general, if the overhaul cost remains constant and we increase the inventory cost, the overhaul zone $A^{\prime}$ decreases. Moreover, it is readily observed in Figure 11 that the repair/overhaul policy is very sensitive to variations of the inventory cost.

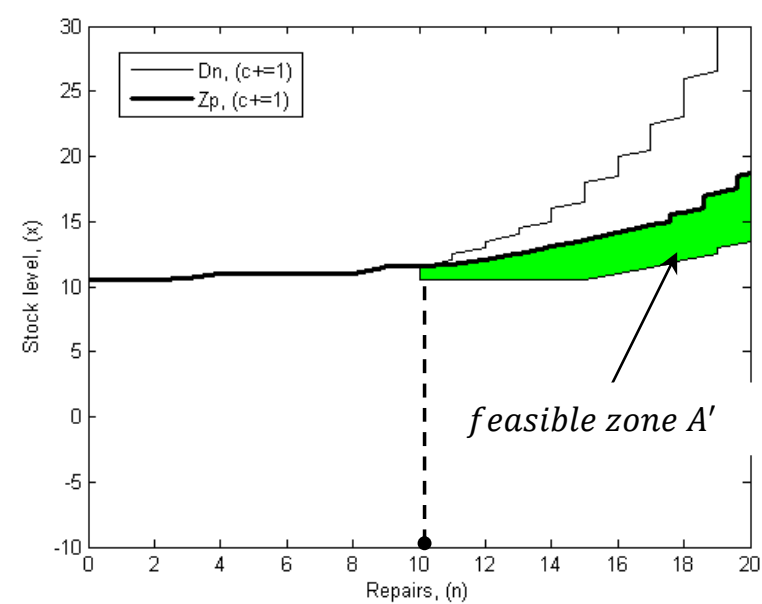

a) $c^{+}=1$

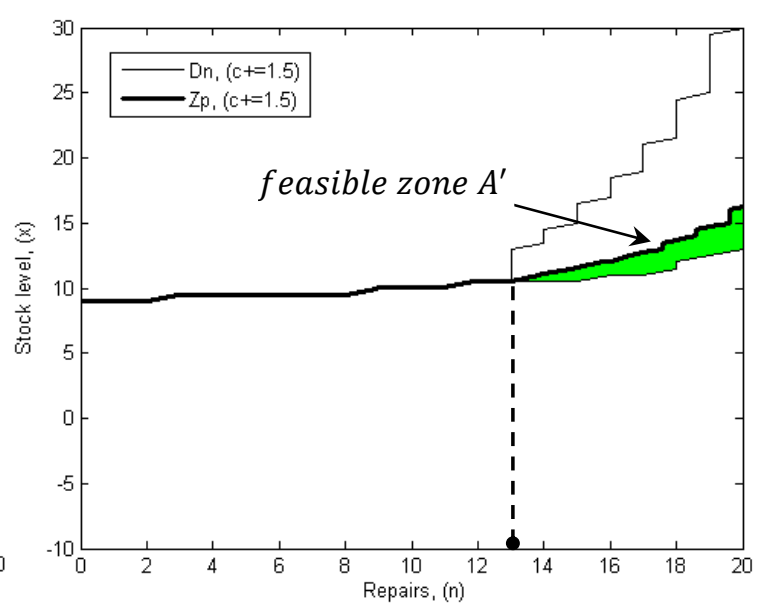

b) $c^{+}=1.5$

Figure 11: Variation of the inventory cost and its effect on the repair/overhaul policy 


\subsection{Variation of the backlog cost}

Our next step in the sensitivity analysis involves the backlog cost $c^{-}$, To examine this parameter, we analyze the production threshold $Z_{p 3}^{*}(\cdot)$ applied in the operational states $O P_{3}^{n}$. From the results of Figure 12, we observe that when the backlog cost is $\mathrm{c}^{-}=100$, the production threshold has an initial value close to 8 , which grows progressively as the number of repairs increases. When the backlog cost increases to $c^{-}=200$, the optimal stock level has an initial value close to 10 , and it follows a parallel trajectory to the previous case. If we increase the backlog cost to $c^{-}=300$, the production threshold grows even more, to a value close to 13 , and the observed trend is parallel to the other cases. These results tell us that by increasing the backlog cost we increase the production threshold, because with a high backlog cost, product shortages are so severely penalized that, higher amounts of products are allowed to be maintained in order to satisfy the demand.

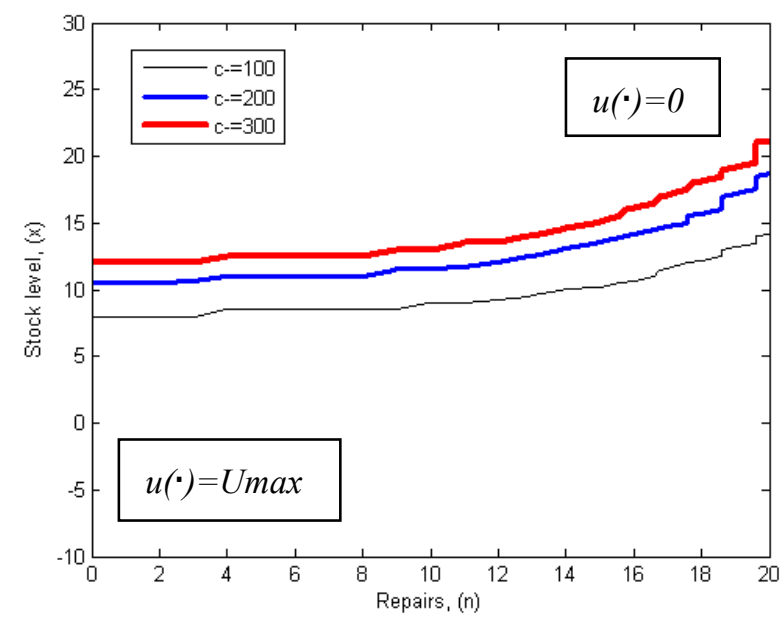

Figure 12: Variation of the backlog cost and its effect on the production threshold

To complement the analysis of the backlog cost, we examine its effect on the repair/overhaul policy. This variation is presented in Figure 13, where it was compared two cases. In analyzing such scenarios, we notice that when the backlog cost is low, for example $c^{-}=150$, the overhaul is less recommended, meanwhile more repairs are performed. Moreover, the minimum number of repairs needed before considering the possibility of the overhaul is $n^{*}=14$, as observed in the repair/overhaul trace $D_{n}\left({ }^{\circ}\right)$. When the backlog cost increases to $c^{-}=200$, the overhaul zone grows, and the number of repairs thus decreases to $n^{*}=7$. The intuition behind this result is that, a variation of the backlog cost is tied directly to the size of the overhaul zone $A^{\prime}$. If the overhaul cost is constant and the backlog cost increases, then the overhaul zone $A^{\prime}$ increases accordingly as well. In the results of Figure 13, it can clearly be seen that changes in the backlog cost influence the repair/overhaul policy. Generally, the effect of the backlog cost on the repair/overhaul policy is the inverse of the effect of the inventory cost. 


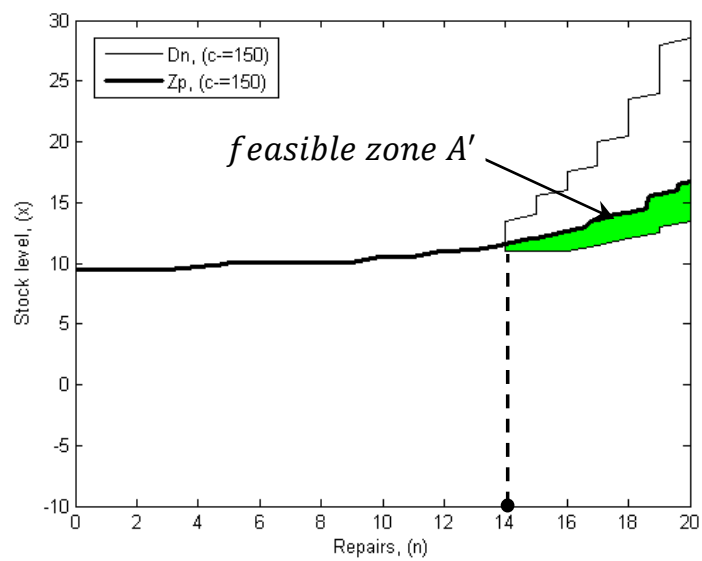

a) $c^{-}=150$

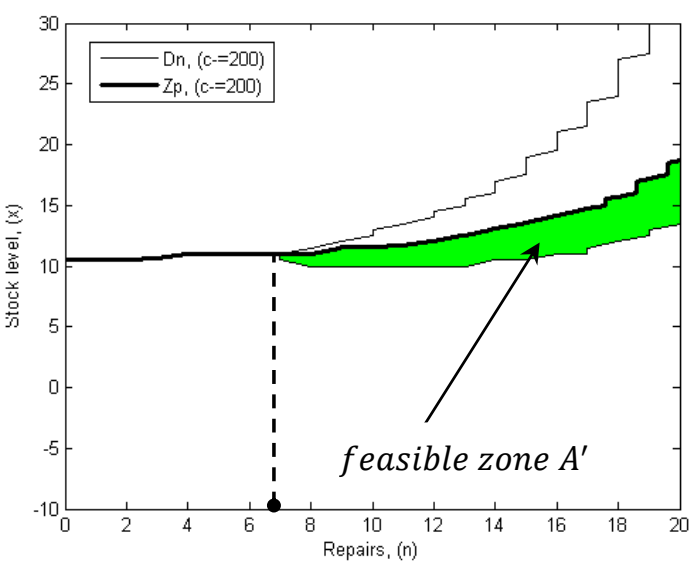

b) $c^{-}=\mathbf{2 0 0}$

Figure 13: Variation of the backlog cost and its effect on the repair/overhaul policy

\subsection{Variation of the overhaul cost}

We shall proceed with the sensitivity analysis, by discussing the overhaul cost $c_{0}$. Figure 14 is intended to analyze the repair/overhaul trace $D_{n}(\cdot)$ for two different values $c_{o}=600$ and 670. From the obtained results, we notice that when the overhaul cost is low, for instance $c_{o}=600$, the overhaul is more recommended. Additionally, the minimum number of repairs needed before considering the possibility of the overhaul is defined as $n^{*}=8$, as indicated in the repair/overhaul trace of Figure 14a. If we increase the cost to $c_{o}=670$, we see a modification in the maintenance trace, and consequently, the overhaul is less recommended. This increases the number of repairs to $n^{*}=14$, as presented in Figure 14b. These results lead us to the argument that the variation of the overhaul cost has a strong effect on the repair/overhaul trace $D_{n}(\cdot)$, and therefore it is evident that, the smaller is the value of the overhaul cost, the more extensive is the overhaul zone $A^{\prime}$. Furthermore, we observe that with low levels of quality deterioration, given by low number of repairs, the overhaul is not recommended. This activity is performed only when the rate of defectives is high enough to justify its expensive cost. With respect to the production policy, the overhaul cost has not shown any effect, since the production threshold in both figures is the same.

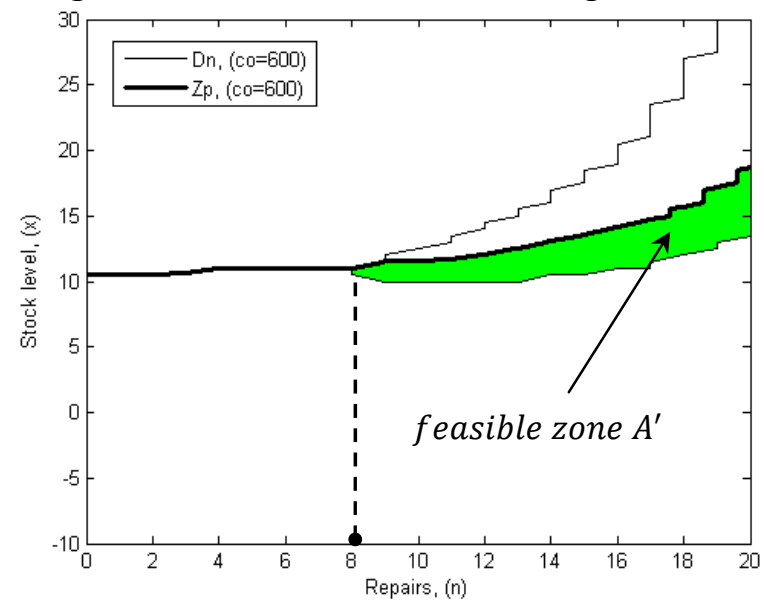

a) $c_{o}=600$

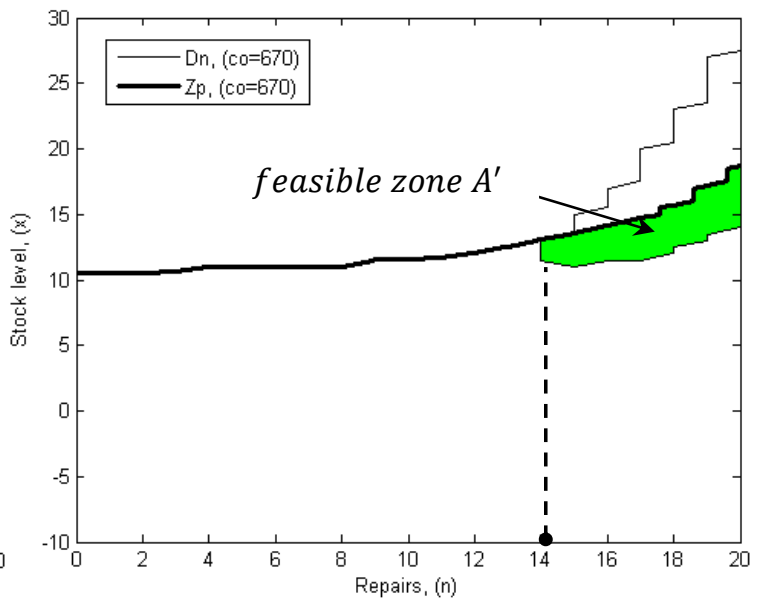

b) $c_{o}=670$

Figure 14: Variation of the overhaul cost and its effect on the repair/overhaul policy 


\subsection{Variation of the repair cost}

To complete the sensitivity analysis, we discuss the variation of the repair cost $c_{r}$, for two cost scenarios, $c_{r}=19$ and 21, as presented in Figure 15. We begin the analysis when the repair cost has a low value $c_{r}=19$; in this case, the overhaul zone $A^{\prime}$ is limited to a small area in the computational domain. The repair/overhaul trace of Figure 15a implies that the minimum number of repairs needed before considering the possibility of the overhaul is set to $n^{*}=13$. Since the repair cost is low, it is recommended to perform fewer overhauls. When the repair cost is $c_{r}=21$, the repair/overhaul trace $D_{n}\left({ }^{\circ}\right)$ varies notably, and the area for the overhaul increases, as a result of which the number of repairs decreases to $n^{*}=7$, as observed in Figure $15 \mathrm{~b}$. These results amount to the observation that the repair/overhaul policy is highly sensitive to the repair cost, since only a very small variation in this parameter is needed to influence the optimal repair/overhaul policy. We notice that by decreasing the repair cost, the zone for performing the overhaul decreases as well. With respect to the effect on the production policy, the repair cost has not reported any influence, since the production threshold in both figures is similar.

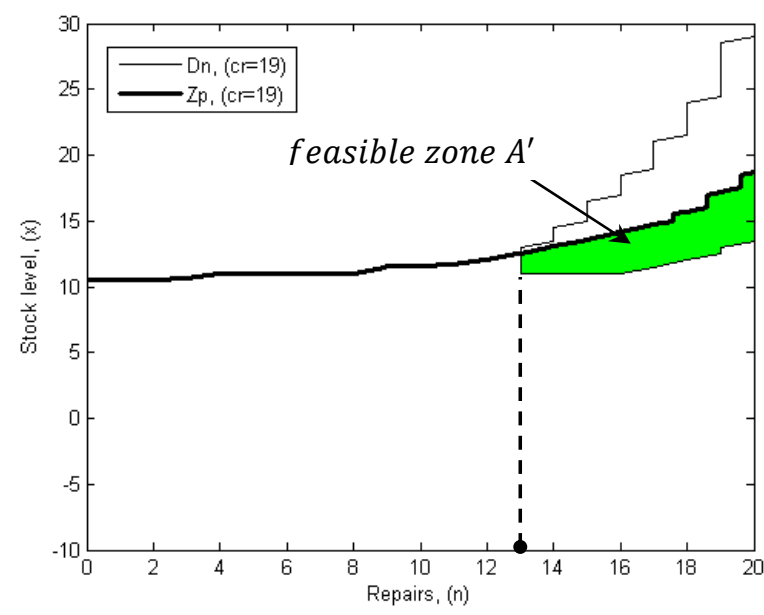

a) $c_{r}=19$

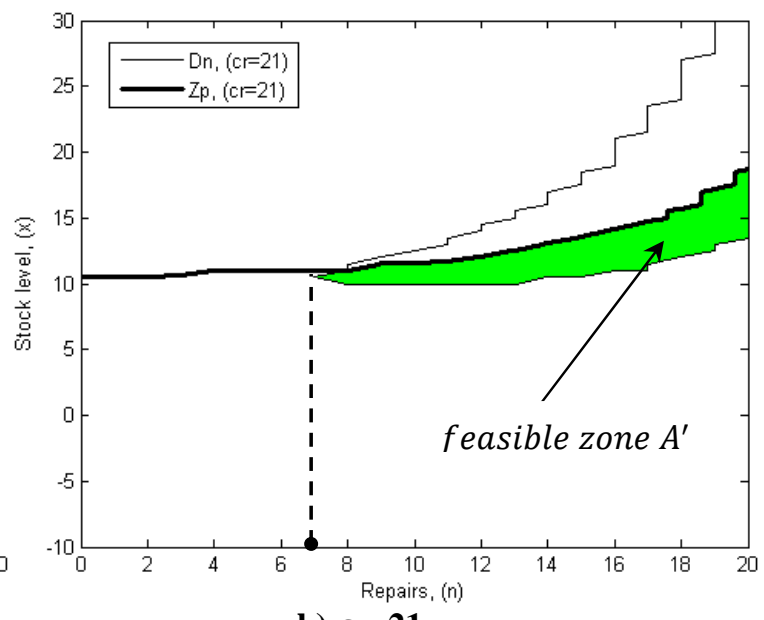

b) $c_{r}=21$

Figure 15: Variation of the repair cost and its effect on the maintenance policy

From the numerical results presented thus far, in this sensitivity analysis, we observe that the traditional production control policy, which consists in maintaining a certain amount of products to hedge against breakdowns, is modified by the presence of the quality deterioration phenomenon. Considering that the machine deteriorates, it implies a policy with several critical thresholds, which increase from one repair to the next, and which also increases between the operational states of the aging process. From the sensitivity analysis, we can conclude that the structure of the obtained control policy is maintained, and this condition yields to the possibility to characterize the optimal policy with the control factors $Z_{p i}^{*}(\cdot)$ and $D_{n}^{*}(\cdot)$. These factors allow the development of a parameterized control policy for simultaneous production planning and the repair/overhaul switching strategy, which seeks to operate the manufacturing system more efficiently at an optimal cost. 


\section{Conclusions}

In this research, we integrated quality issues in the determination of control policies, and this implied that a repair/overhaul planning problem is combined with a production control problem for a manufacturing system subject to deterioration. The effect of the deterioration phenomenon on the machine is reflected in the quality of the parts produced, where the rate of defectives increases due to the combination of two factors: the wear of the machine given by an aging process, and human interventions, tied to worse repairs. We observed that the stock level required to hedge against breakdowns increases with the number of repairs and the evolution in the set of operational states. We found that the performance of overhaul activities depends on the stock level and the number of repairs. Since we integrate the history of the production system into, the number of repairs and the use of multiple operational states, a semi-Markov model was developed. A numerical example was considered to illustrate the utility of the proposed approach, and a sensitivity analysis was conducted to confirm the structure of the control policy. The obtained policy resulted in an interesting alternative for controlling the manufacturing system at the operational level, compared to other works that does not consider quality deterioration. Finally, the assessment of the proposed model shows that quality deterioration has a considerable effect on the production and repair/overhaul policy.

\section{References}

[1] Akella, R., Kumar, P.R., 1986, Optimal control of production rate in a failure prone manufacturing system, IEEE Transactions on Automatic Control, vol. AC31, pp. 116-126.

[2] Besterfield, D.H., 2009, Quality Control, Prentice Hall, Eight edition, Upper Saddle River, New Jersey.

[3] Bielecki, T., Kumar, P.R., 1988, Optimality of zero inventory policies for unreliable manufacturing systems, Operations Research, vol. 36, pp. 532-541, Jul.-Aug. 1988.

[4] Bonvik, A.,M., Dallery, Y., Gershwin, S.B., 2000, Approximate analysis of production systems operated by a CONWIP/finite buffer hybrid control policy, International Journal of Production Research, 38:13, pp. 2845-2869.

[5] Colledani, M., Tolio, T., 2006, Impact of Quality Control on Production System Performance, CIRP Annals - Manufacturing Technology, vol. 55, No. 1, pp. 453456.

[6] Colledani, M., Tolio T., 2009, Performance evaluation of production systems monitored by statistical process control and off-line inspections. International Journal of Economics, 120, pp. 348-367. 
[7] Colledani, M. and Tolio, T., 2011, Integrated analysis of quality and production logistics performance in manufacturing lines, International Journal of Production Research, 49:2, pp. 485-518.

[8] Chelbi, A., Ait-Kadi, D., 2004, Analysis of a production/inventory system with randomly failing production unit submitted to regular preventive maintenance. European Journal of Operational Research, No.156, pp. 712-718.

[9] Dahane, M., Rezg, N., Chelbi, A., 2012, Optimal production plan for a multiproducts manufacturing system with production rate dependent failure rate, International Journal of Production Research, 50:13, pp. 3517-3528.

[10] Dehayem Nodem F.I., Kenne, J.P., Gharbi, A., 2011a, Production planning and repair/replacement switching policy for deteriorating manufacturing systems, International Journal of Advance Manufacturing Technology, No. 57, pp. 827840.

[11] Dehayem Nodem F.I., Gharbi, A., Kenne, J.P., 2011b, Preventive maintenance and replacement policies for deteriorating productions systems subject to imperfect repairs, International Journal of Production Research, No. 49, pp. 3543-3563.

[12] Gershwin, S.B., 2002, Manufacturing Systems Engineering, Massachusetts Institute of Technology, Second private printing, Cambridge, Massachusetts, USA.

[13] Gharbi, A., Kenne, J. P., 2003, Optimal production control problem in stochastic multiple-product multiple-machine manufacturing systems, IEEE Transactions, vol. 35 , No. 10, pp. 941-52.

[14] Hajji, A., Gharbi, A., Kenne, J.P., 2009, Joint replenishment and manufacturing activities control in a two stage unreliable supply chain, International Journal of Production Research, 47:12, pp. 3231-3251.

[15] Inman, R.R., Blumenfeld, D.E., Huang, N., 2003, Designing production systems for quality: research opportunities from an automotive industry perspective, International Journal of Production Research, 41:9, pp. 1953-1971.

[16] Kenne, J.P., Boukas, E.K., Gharbi, A., 2003, Control of production and corrective maintenance rates in a multiple-machine, multiple-product manufacturing system. Mathematical and Computer modeling 38 (3-4), 351-365.

[17] Kim, J., 2005, Integrated Quality and Quantity Modeling of a Production Line. MIT, Ph.D. Thesis dissertation.

[18] Kim, J., Gershwin, S., 2005, Integrated quality and quantity modeling of a production line, OR Spectrum 27, pp. 287-314.

[19] Kim, J., Gershwin, S., 2008, Analysis of long flow lines with quality and operational failures, IIE Transactions, 40, pp. 284-296. 
[20] Kushner, H.J. and Dupuis, P.G., 1992, Numerical Methods for Stochastic Control Problems in Continuous Time, (Springer, New York, NY).

[21] Lam, Y. and Chan, S.K., 1998, Statistical inference for geometric processes with lognormal distribution, Computational Statistics \& Data Analysis, 27 pp. 99-12.

[22] Lam, Y., Zhu, L.X., Chan, J.S.K., Liu, Q., 2004, Analysis of data from a series of events by a geometric process model, Acta Mathematicae Applicatae 20, pp. 263282.

[23] Lam, Y., 2007, A geometric process maintenance model with preventive repair, European Journal of Operation Research, 182, pp. 806-819.

[24] Leung, H., 2001, Optimal replacement policies determined using arithmeticogeometric processes, Engineering Optimization, 33:4, pp. 473-484.

[25] Love, C.E. and Zitron, M.A., 1998, An SMDP approach to optimal repair/replacement decisions for systems experiencing imperfect repairs, Journal of Quality in Maintenance Engineering, vol. 4, No. 2, pp. 131-149.

[26] Love, C.E., Zhang, Z.G., Zitron, M.A., Guo, R., 2000, A discrete semi-Markov decision model to determine the optimal repair/replacement policy under general repairs, European Journal of Operational Research, 125 pp. 398-409.

[27] Njike, A.N., Pellerin, R., Kenne, J.,P., 2009, Simultaneous control of maintenance and production rates of a manufacturing system with defective products. Journal of Intelligent Manufacturing 10845, article 354.

[28] Pham, H. and Wang H., 1996, Imperfect maintenance, European Journal of Operation Research, No. 94, pp. 425-438.

[29] Radhoui, M., Rezg, N., Chelbi, A., 2009, Integrated model of preventive maintenance, quality control and buffer sizing for unreliable and imperfect production systems, International Journal of Production Research, vol. 47, No. 2, pp. 389-402.

[30] Rezg, N., Dellagi, S., Chelbi, A., 2008, Joint optimal inventory control and preventive maintenance policy, International Journal of Production Research, 46, pp. 5349-5365.

[31] Rishel, R., 1975, Dynamic programming and minimum principles for systems with jump Markov disturbances, SIAM Journal on Control 13, pp. 338-371.

[32] Sharifnia, A., 1988, Production Control of a Manufacturing System with multiple Machine States, IEEE Transactions on Automatic Control, vol. 22, No. 7. 